\title{
Strong Magnetic Fields, Dirichlet Boundaries, and Spectral Gaps
}

\author{
Rainer Hempel ${ }^{1}$, Ira Herbst ${ }^{2,3}$ \\ Erwin Schrödinger International Institute for Mathematical Physics, Pasteurgasse 4/7, A-1090 \\ Vienna, Austria
}

Received: 1 January 1994/in revised form: 11 May 1994

\begin{abstract}
We consider magnetic Schrödinger operators

$$
H(\lambda \vec{a})=(-i \nabla-\lambda \vec{a}(x))^{2}
$$

in $L_{2}\left(\mathbf{R}^{n}\right)$, where $\vec{a} \in C^{1}\left(\mathbf{R}^{n} ; \mathbf{R}^{n}\right)$ and $\lambda \in \mathbf{R}$. Letting $M=\{x ; B(x)=0\}$, where $B$ is the magnetic field associated with $\vec{a}$, and $M_{\vec{a}}=\{x ; \vec{a}(x)=0\}$, we prove that $H(\lambda \vec{a})$ converges to the (Dirichlet) Laplacian on the closed set $M$ in the strong resolvent sense, as $\lambda \rightarrow \infty$, provided the set $M \backslash M_{\vec{a}}$ has measure zero.

In various situations, which include the case of periodic fields, we even obtain norm resolvent convergence (again under the condition that $M \backslash M_{\vec{a}}$ has measure zero). As a consequence, if we are given a periodic field $B$ where the regions with $B=0$ have non-empty interior and are enclosed by the region with $B \neq 0$, magnetic wells will be created when $\lambda$ is large, opening up gaps in the spectrum of $H(\lambda \vec{a})$.

We finally address the question of absolute continuity of $H(\vec{a})$ for periodic $\vec{a}$.
\end{abstract}

\section{Introduction}

While the resolvent-limit of the Schrödinger operators $-\Delta+\lambda \chi_{\Omega}$, as $\lambda \rightarrow \infty$, has been thoroughly studied (cf., e. g., Herbst and Zhao [HZh], Arendt and Batty [AB]), it seems that little - if anything at all - is known about the corresponding situation of magnetic perturbations of the Laplacian,

$$
H(\lambda \vec{a})=(-i \nabla-\lambda \vec{a})^{2},
$$

as $\lambda \rightarrow \infty$, where $\vec{a}$ is a vector potential on $\mathbf{R}^{n}$ of class $C^{1}$ or $C^{2}$. Recalling that, for $\Omega$ an open subset of $\mathbf{R}^{n}$, the strong resolvent limit of $-\Delta+\lambda \chi_{\Omega}$ is given by $-\Delta_{M}$,

\footnotetext{
1 Department of Mathematics, Univ. of Alabama at Birmingham, Birmingham, AL 35294-1170, USA. Part of this work was done while on leave from Mathemat. Institut, Univ. München, Theresienstr. 39, D-80333 München, Germany.

2 On leave from Univ. of Virginia, Charlottesville, VA 22903, USA.

3 Research partially supported by USNSF grant DMS 9307147.
} 
the Dirichlet Laplacian on the closed set $M=\Omega^{C}$ (cf. Sect. 1 for details), one may wonder whether $H(\lambda \vec{a})$ will also converge in strong (or even norm) resolvent sense to a Laplacian on a suitable domain. In the case of magnetic perturbations, however, there are two natural domains, one connected with the vector potential $\vec{a}$ and the other related to the magnetic field, namely the set

$$
M_{\vec{a}}=\left\{x \in \mathbf{R}^{n} ; \vec{a}(x)=0\right\},
$$

where the vector potential $\vec{a}$ vanishes, and the set

$$
M=\left\{x \in \mathbf{R}^{n} ; d \vec{a}=0\right\},
$$

where the magnetic field $B=d \vec{a}=\sum B_{i j}(x) d x_{i} \wedge d x_{j}$ is zero (here we think of $d \vec{a}$ as the exterior derivative of the 1 -form associated with $\vec{a})$. In general, the sets $M$ and $M_{\vec{a}}$ will be different, but we always have meas $\left(M_{\vec{a}} \backslash M\right)=0$ (see [GT; Lemma 7.7]). Strangely enough, there are (quite different) intuitive reasons why the resolvent $(H(\lambda \vec{a})+1)^{-1}$ should tend to zero on the subspace $L_{2}(\Omega)$ as well as on the subspace $L_{2}\left(\Omega_{\vec{a}}\right)$, where $\Omega=M^{C}$ and $\Omega_{\vec{a}}=M_{\vec{a}}^{C}$ : the fact that we have a non-zero magnetic field on $\Omega$ easily translates into a lower bound for the quadratic form on $C_{c}^{\infty}(\Omega)$, which diverges as $\lambda \rightarrow \infty$, while, on the other hand, the vector potential $\vec{a}$ doesn't vanish on $\Omega_{\vec{a}}$ so that the (classical) symbol $\left((\xi-\lambda \vec{a}(x))^{2}+1\right)^{-1}$ will tend to 0 on $\Omega_{\vec{a}}$, uniformly for $|\xi| \leqq R$. Of course, the classical symbol does not tell the full truth, and quantum mechanics introduces some non-locality. As it turns out, $(H(\lambda \vec{a})+1)^{-1}$ can not be expected to converge to $\left(-\Delta_{M}+1\right)^{-1}$ if the sets $M$ and $M_{\vec{a}}$ differ too much (cf. Theorem 4.1 and the discussion in Sect. 2).

Our results can be summarized as follows:

(1) We find that $(H(\lambda \vec{a})+1)^{-1}$ converges strongly to the 0-operator in $L_{2}\left(\mathbf{R}^{n}\right)$ if the vector potential $\vec{a} \in C^{2}\left(\mathbf{R}^{n} ; \mathbf{R}^{n}\right)$ is non-zero almost everywhere (cf. Theorem 2.1).

(2) On the other hand, if $M \backslash M_{\vec{a}}$ has measure 0 (so that the sets where $\vec{a}$ and $B$ vanish are the same, up to a set of measure 0$)$, then the resolvent of $H(\lambda \vec{a})$ will converge strongly to the resolvent of $-\Delta_{M}$, the Dirichlet Laplacian on the set $M$ (cf. Theorem 2.3). We say that a vector potential satisfies Condition $S_{\vec{a}}$ if $M \backslash M_{\vec{a}}$ has measure 0 . Under this condition, we obtain a type of convergence which is intermediate between strong and norm resolvent convergence in the sense that convergence is uniform in the gauge, in particular uniform with respect to translations in momentum space. It is shown in Theorem 4.1 that this type of convergence can only occur if $M \backslash M_{\vec{a}}$ has empty interior, and we thus see that Condition $S_{\vec{a}}$ is (almost) necessary and sufficient for having strong resolvent convergence, uniform in the gauge.

It is an interesting topological and analytical problem to determine general conditions under which - given a smooth $B$ with $d B=0$ defined on $\mathbf{R}^{n}$ - we can find a smooth $\vec{a}$ such that $B=d \vec{a}$ and $M \backslash M_{\vec{a}}$ has measure zero. Roughly speaking, given an $\vec{a}^{\prime}$ with $d \vec{a}^{\prime}=B$, we can define a function $f$ in the region where $B=0$ by a line integral of $\vec{a}^{\prime}$ if this region is simply connected, or if, more generally, a zero-flux condition holds (see Sect. 4). We may then extend $f$ smoothly to $\mathbf{R}^{n}$ ([GT], [St]) and define $\vec{a}=\vec{a}^{\prime}-\nabla f$. Then (roughly speaking) $\vec{a}=0$ on $M$.

(3) Under suitable stronger assumptions we may even obtain norm resolvent convergence to the Dirichlet Laplacian on $M$. Our main results in this direction, given in the Corollaries 3.3, 3.4 and Theorem 3.9, cover the cases where $\Omega_{\vec{a}}$ has compact closure, or where we have some type of spatial uniformity of the data. 
Our results apply in particular to all periodic fields $B=d \vec{a}$ with the property that meas $\left(M \backslash M_{\vec{a}}\right)=0$, where we find that $H(\lambda \vec{a})$ converges in norm resolvent sense to the Dirichlet Laplacian on $M$. Therefore, we are able to construct situations where gaps in the spectrum of $H(\lambda \vec{a})$ open up as $\lambda \rightarrow \infty$ (cf. Corollary 3.10 and Example 3.11). This result lies somewhat in between the classical results concerning the existence of spectral gaps for periodic Schrödinger operators (cf., e. g., [RSIV]), and the more recent results of Davies and Harrell [DH] and Hempel [He] on the existence of gaps for periodic Laplace-Beltrami operators and for periodic divergence type operators, respectively. Finding examples of periodic magnetic Laplacians with spectral gaps was the starting point and the basic motivation of the present work. In a more general context which includes the case of periodic magnetic fields, Brüning and Sunada [BrS1, BrS2] have recently obtained some basic results on the structure of the spectrum; cf. the remarks following Corollary 3.10 .

It is well-known that a non-zero constant field in 2-dimensions leads to spectral gaps. One should note that what we consider here is genuinely different in the sense that in our examples the gaps are produced by a periodic array of magnetic "wells" (where the field is zero) which are separated from one another by magnetic "barriers" which make tunnelling from one well into any other well more and more difficult, as the coupling $\lambda$ increases.

(4) Finally, in the case of a periodic vector potential $\vec{a}$, it is natural to ask whether the spectrum will be purely absolutely continuous. Following the proof of Thomas [Th] we are able to prove this for small coupling (Theorem 3.12), but must leave the question open in general.

To conclude the introduction, let us briefly indicate some of the main steps leading to the above results. Apart from the somewhat special argument used in obtaining Theorem 2.1, all our results on resolvent convergence assume condition $S_{\vec{a}}$ and use the following ideas:

Let $\Omega=M^{C}$ and exhaust $\Omega$ by a sequence of open sets $\Omega_{n} \uparrow \Omega$ in such a way that Theorem 2.9 of Avron, Herbst and Simon [AHS] gives a uniform lower bound for the quadratic form on $\Omega_{n}$,

$$
(H(\lambda \vec{a}) \varphi, \varphi) \geqq c_{n} \lambda\|\varphi\|^{2}, \quad \varphi \in C_{c}^{\infty}\left(\Omega_{n}\right),
$$

with a positive constant $c_{n}$. From this we infer the resolvent estimate of Lemma 2.3,

$$
\left\|(H(\lambda \vec{a})+1)^{-1} \chi_{\Omega_{n}}\right\| \leqq c_{n}^{\prime} \lambda^{-1 / 2}
$$

which is then used to "enhance" the convergence of resolvents by adding in multiples of $\chi_{\Omega_{n}}$. In fact, we show in Lemma 2.4 that we can find $n(\lambda)$ tending to $\infty$ (as $\lambda \rightarrow \infty$ ) such that

$$
\left\|(H(\lambda \vec{a})+1)^{-\uparrow}-\left(H(\lambda \vec{a})+n(\lambda) \chi_{\Omega_{n(\lambda)}}+1\right)^{-1}\right\| \rightarrow 0, \quad \lambda \rightarrow \infty .
$$

In order to estimate the difference $(H(\lambda \vec{a})+1)^{-1} f-\left(-\Delta_{M}+1\right)^{-1} f$, for a given $f \in L_{2}$, we then only need to control $\left(H(\lambda \vec{a})+n(\lambda) \chi_{\Omega_{n(\lambda)}}+1\right)^{-1} f-\left(-\Delta_{M}+\right.$ $1)^{-1} f$. Using the Feynman-Kac-Itô formula, it is easily seen that this term is dominated by an analogous expression without the $\lambda \vec{a}$-term. Thus we have effectively reduced the convergence problem for the magnetic case to the more standard convergence problem for $-\Delta+n \chi_{\Omega_{n}}, n \rightarrow \infty$. While strong resolvent convergence of $-\Delta+n \chi_{\Omega_{n}}$ to $-\Delta_{M}$ is always true, we need additional assumptions to obtain norm 
resolvent convergence (keeping in mind that the choice of the sets $\Omega_{n}$ depends on properties of the coefficients $B_{i j}$ ). Typical situations are discussed in Corollaries 3.3, 3.4 and Theorem 3.9, which deal with the cases $\bar{\Omega}$ compact, $\Omega$ a half-space, and $\Omega$ periodic, respectively.

\section{Preliminaries and Notation}

Suppose we are given a vector potential $\vec{a} \in C^{1}\left(\mathbf{R}^{n} ; \mathbf{R}^{n}\right)$ and a bounded, measurable potential $V: \mathbf{R}^{n} \rightarrow \mathbf{R}$. In the Hilbert space $L_{2}\left(\mathbf{R}^{n}\right)$, we then define the Schrödinger operator $H(\vec{a})+V$ as the unique self-adjoint and non-negative operator associated with the (closed) quadratic form

$$
\sum_{j=1}^{n}\left\|-i \partial_{j} u-a_{j} u\right\|^{2}+\int V|u|^{2} d x
$$

defined on the space of functions $u \in L_{2}\left(\mathbf{R}^{n}\right)$ for which $-i \partial_{j} u-a_{j} u \in L_{2}\left(\mathbf{R}^{n}\right)$, for $j=1, \ldots, n$. Here $\partial_{j} u$ denotes the distributional derivative; for $u$ in the form domain, it is clear that these distributional derivatives are locally square integrable. Under the strong assumptions on $\vec{a}$ and $V$ made here, it is easy to see that $C_{c}^{\infty}\left(\mathbf{R}^{n}\right)$ is a form core. (This is actually true in much greater generality; also, it is known that $C_{c}^{\infty}\left(\mathbf{R}^{n}\right)$ is an operator core, but we will not need this fact; cf. [CFrKS, S3] etc.) Formally, the operator $H(\vec{a})+V$ may be written as $(-i \nabla-\vec{a})^{2}+V$; occasionally, we will also use the notation $\vec{p}$ instead of $-i \nabla$.

We next wish to discuss a special case of the results given in Herbst and Zhao [HZh] (cf. also Hedberg [H]) on the strong resolvent limit of $-\Delta+n \chi_{\Omega}$, for $n \rightarrow \infty$, where $\Omega$ is an open set. Letting $M=\Omega^{C}$, we introduce the Sobolev space

$$
\mathscr{H}^{1}(M)=\left\{\varphi \in W_{2}^{1}\left(\mathbf{R}^{n}\right) ; \varphi(x)=0 \text { a. e. in } \Omega\right\},
$$

consisting of all (equivalence classes of) functions $\varphi \in W_{2}^{1}\left(\mathbf{R}^{n}\right)$ which satisfy $\varphi(x)=0$ for a. e. $x \in \Omega$; here $W_{2}^{1}\left(\mathbf{R}^{n}\right)$ is the usual Sobolev space consisting of all $L_{2}$-functions $u$ such that $\partial_{j} u \in L_{2}, j=1, \ldots, n$. (We warn the reader that the space $\mathscr{H}^{1}(M)$ is denoted by $\tilde{H}_{0}^{1}(M)$ in $[\mathrm{HZh}]$.) Then $-\Delta_{M}$ is defined as the unique non-negative self-adjoint operator associated with the quadratic form $\int|\nabla \varphi|^{2} d x$ defined on $\mathscr{H}^{1}(M)$. It is immediate from our definition that the operators $-\Delta_{M}$ and $-\Delta_{M^{\prime}}$ are the same if $M, M^{\prime}$ are closed sets with meas $\left(M \triangle M^{\prime}\right)=0$. We call $-\Delta_{M}$ the Dirichlet Laplacian on the set $M$. Under mild regularity conditions on the boundary of $M$ (which are even weaker than the segment condition) it also follows that $-\Delta_{M}$ coincides with the usual Dirichlet Laplacian on the interior of $M$; see [HZh].

We write $-\Delta$ to denote the unique self-adjoint extension of $-\Delta \mid C_{c}^{\infty}\left(\mathbf{R}^{n}\right)$. Using the notation $\Omega_{n} \uparrow \Omega$ to indicate that $\Omega_{n} \subset \Omega_{n+1}$ and $\cup \Omega_{n}=\Omega$, we have the following result on strong resolvent convergence for the sequence of operators $-\Delta+n \chi_{\Omega_{n}}$.

1.1. Lemma. Suppose $\Omega_{n} \uparrow \Omega$, where $\Omega, \Omega_{n}$ are open subsets of $\mathbf{R}^{n}$. Let $M=\Omega^{C}$ and let $-\Delta_{M}$ denote the Dirichlet Laplacian on $M$, as defined above. Then, as $n \rightarrow \infty$,

$$
-\Delta+n \chi_{\Omega_{n}} \rightarrow-\Delta_{M} \text { in strong resolvent sense }
$$


Remark. The operator $-\Delta_{M}$ acts in the Hilbert space $\overline{\mathscr{H}^{1}(M)}$, where the bar denotes the closure in $L_{2}\left(\mathbf{R}^{n}\right)$. In general, this space will be smaller than $L_{2}(M)$, but it is shown in $[\mathrm{HZh}]$ that there always exists a subset $M^{*} \subset M$ such that $\overline{\mathscr{H}}(\bar{M})=$ $L_{2}\left(M^{*}\right)$. Hence the statement of Lemma 1.1 has to be interpreted in the sense that $\left(-\Delta+n \chi_{\Omega_{n}}+1\right)^{-1}$ converges to the direct sum of the operator $\left(-\Delta_{M}+1\right)^{-1}$, acting in $L_{2}\left(M^{*}\right)$, and the zero-operator on $L_{2}\left(M^{*}\right)^{\perp}$ (cf. Weidmann [W] or Simon [S2] for a discussion of strong resolvent convergence in such cases). E. g., in the extreme case where $M$ (or $M^{*}$ ) is a set of measure zero, the statement of Lemma 1.1 means that the resolvent of $-\Delta+n \chi_{\Omega_{n}}$ converges strongly to zero.

Similarly, the operators $e^{t \Delta_{M}}$ may have to be complemented by the zero-operator on the subspace $L_{2}\left(M^{*}\right)^{\perp}$, for $t>0$. Note that, in general, $\left(e^{t \Delta_{M}} ; t \geqq 0\right)$ will not form a $C_{0}$-semigroup in the Hilbert space $L_{2}\left(\mathbf{R}^{n}\right)$.

Proof of Lemma 1.1. The quadratic forms associated with the operators $-\Delta+n \chi_{\Omega_{n}}$ have constant domain $W_{2}^{1}\left(\mathbf{R}^{n}\right)$ and are monotonically increasing. Defining $T$ to be the self-adjoint and non-negative operator associated with the quadratic form $\|\nabla u\|^{2}$ with domain given by the space of all functions $u \in W_{2}^{1}\left(\mathbf{R}^{n}\right)$ for which sup $n\left\|\chi_{\Omega_{n}} u\right\|^{2}$ is finite, it follows (see [S2, HZh or W]) that $-\Delta+n \chi_{\Omega_{n}}$ converges to $T$ in strong resolvent sense. It is easily seen that $T=-\Delta_{M}$, and the result follows.

We now use the above lemma to derive a Feynman-Kac representation for the operators $\mathrm{e}^{t \Delta_{M}}$, for $t>0$, associated with $-\Delta_{M}$. Here and in the sequel we will write $\mathbf{E}_{x}$ to denote expectation with respect to Wiener measure on the set of continuous Brownian paths $\omega$ starting at the point $x$ at time $t=0$.

1.2. Lemma. ([HZh]) Let $M$ be a closed subset of $\mathbf{R}^{n}$ and let $-\Delta_{M}$ as above. For $f \in C_{c}^{\infty}\left(\mathbf{R}^{n}\right)$ and $t>0$ we have

$$
\left(e^{t \Delta_{M}} f\right)(x)=\mathbf{E}_{x}\left(\chi_{\{\omega, \omega(s) \in M, 0 \leqq s \leqq t\}} f(\omega(t))\right), \text { for a. } e . x \in \mathbf{R}^{n} .
$$

Proof. Let $\Omega=M^{C}$. By the usual Feynman-Kac formula we know that for any $f \in C_{c}^{\infty}\left(\mathbf{R}^{n}\right)$

$$
\left(\mathrm{e}^{-t\left(-\Delta+n \chi_{\Omega}\right)} f\right)(x)=\mathbf{E}_{x}\left(\mathrm{e}^{-n \int_{0}^{t} \chi_{\Omega}(\omega(s)) d s} f(\omega(t))\right) .
$$

Here the function $G_{n}$, defined on the space of continuous functions $\omega: \mathbf{R} \rightarrow \mathbf{R}^{n}$ by

$$
G_{n}(\omega):=\mathrm{e}^{-n \int_{0}^{t} \chi_{\Omega}(\omega(s)) d s}
$$

is monotonically decreasing in $n$ and converges to the characteristic function of the set $\{\omega ; \omega(s) \in M, s \in[0, t]\}$. In order to prove this, suppose that $\omega\left(s_{0}\right) \in \Omega$, for some $s_{0} \in[0, t]$. Since $\omega$ is continuous, it follows that $\omega(s) \in \Omega$ for $s$ in some neighborhood of $s_{0}$. Thus $\int_{0}^{t} \chi_{\Omega}(\omega(s)) d s>0$, so that $G_{n}(\omega) \rightarrow 0$ for all paths $\omega$ meeting $\Omega$. Conversely, if $\omega(s) \in \Omega^{C}$, for all $s \in[0, t]$, then $G_{n}(\omega)=1$ for all $n$.

Now it follows by dominated convergence that the RHS of (1.2) tends to the expression on the RHS of (1.1), while, on the LHS, Lemma 1.1 implies that there exists a subsequence of $n$ 's such that $\mathrm{e}^{-t\left(-\Delta+n \chi_{\Omega}\right)} f \rightarrow \mathrm{e}^{t \Delta_{M}} f$, for a. e. $x$, and we are done.

We finally employ the Feynman-Kac-Itô formula ([S3]) to eliminate the vector potential $\vec{a}$ from our further estimates. 
1.3. Lemma. Let $\vec{a} \in C^{1}\left(\mathbf{R}^{n} ; \mathbf{R}^{n}\right), \quad M_{\vec{a}}=\{x ; \vec{a}(x)=0\}, \quad M=\{x ; B(x)=d \vec{a}(x)=$ $0\}$, and suppose $M \backslash M_{\vec{a}}$ has measure zero. Suppose $\tilde{\Omega} \subset \Omega$ is given, and let $\tilde{\chi}=\chi_{\tilde{\Omega}}$. We then have for $t>0, n \geqq 0$, and $f \in L_{2}\left(\mathbf{R}^{n}\right)$,

$$
\left\|\left(e^{-t(H(\vec{a})+n \tilde{\chi})}-e^{t \Delta_{M}}\right) f\right\| \leqq\left\|\left(e^{-t(-\Delta+n \tilde{\chi})}-e^{t \Delta_{M}}\right)|f|\right\| .
$$

Proof. By the Feynman-Kac-Itô formula and Lemma 1.2, we find for $f \in C_{c}^{\infty}\left(\mathbf{R}^{n}\right)$ that for a. e. $x$,

$$
\begin{aligned}
& \left(\mathrm{e}^{-t(H(\vec{a})+n \tilde{\chi})} f-\mathrm{e}^{t \Delta_{M}} f\right)(x) \\
= & \mathbf{E}_{x}\left\{\mathrm{e}^{i \Phi(\omega, t)}\left(\mathrm{e}^{-n \int_{0}^{t} \tilde{\chi}(\omega(s)) d s}-\chi_{\{\omega ; \omega(s) \in M, 0 \leqq s \leqq t\}}\right) f(\omega(t))\right\},
\end{aligned}
$$

where $\Phi(\omega, t)$ is a real-valued phase factor satisfying $\Phi(\omega, t)=0$ for any $\omega$ that does not leave the set $M_{\vec{a}}$ for $0 \leqq s \leqq t$ (note that we have used the fact that since $\mathscr{H}^{\mathrm{l}}(M)=\mathscr{H}^{\mathrm{l}}\left(M_{\vec{a}}\right)$ we have $\left.\Delta_{M}=\bar{\Delta}_{M_{\vec{a}}}\right)$. It follows that for a. e. $x$

$$
\begin{aligned}
& \left|\left(\mathrm{e}^{-t(H(\vec{a})+n \tilde{\chi})} f-\mathrm{e}^{t \Delta_{M}} f\right)(x)\right| \\
& \quad \leqq \mathbf{E}_{x}\left\{\left(\mathrm{e}^{-n \iint_{0}^{t} \tilde{x}(\omega(s)) d s}-\chi_{\{\omega ; \omega(s) \in M, 0 \leqq s \leqq t\}}\right)|f(\omega(t))|\right\} \\
& =\left(\mathrm{e}^{-t(-\Delta+n \tilde{x})}|f|-\mathrm{e}^{t \Delta_{M}}|f|\right)(x),
\end{aligned}
$$

and we are done.

The quadratic form lower bound of [AHS; Theorem 2.9] is fundamental for most of our results. Since its proof is so simple, we reproduce it here for the reader's convenience.

1.4. Proposition. ([AHS]) Let $\vec{\Pi}=-i \nabla-\vec{a}$, with $\vec{a} \in C^{1}\left(\mathbf{R}^{n} ; \mathbf{R}^{n}\right)$. Then for any $\varphi \in C_{c}^{\infty}\left(\mathbf{R}^{n}\right)$,

$$
\left|\left(\varphi,\left(\partial_{i} a_{j}-\partial_{j} a_{i}\right) \varphi\right)\right| \leqq\left\|\Pi_{i} \varphi\right\|^{2}+\left\|\Pi_{J} \varphi\right\|^{2} .
$$

Proof. Applying the Schwarz inequality to the LHS of the commutation relation

$$
\left[\Pi_{i}, \Pi_{j}\right] \varphi=i\left(\partial_{i} a_{j}-\partial_{j} a_{i}\right) \varphi,
$$

we find that

$$
\left|\left(\varphi,\left(\partial_{i} a_{j}-\partial_{j} a_{i}\right) \varphi\right)\right| \leqq 2\left\|\Pi_{i} \varphi\right\|\left\|\Pi_{j} \varphi\right\| \leqq\left\|\Pi_{i} \varphi\right\|^{2}+\left\|\Pi_{j} \varphi\right\|^{2} .
$$

\section{Strong Resolvent Convergence}

In this section, we study situations where a strong resolvent limit exists.

We first present a simple result on strong resolvent convergence for $\vec{a}$ 's which are non-zero a. e.; here we find that the resolvent of $H(\lambda \vec{a})$ converges strongly to the zero operator, as $\lambda \rightarrow \infty$. Note that the subsequent results in Sects. 2 and 3 are independent of Theorem 2.1. 
2.1. Theorem. Suppose that $\vec{a} \in C^{2}\left(\mathbf{R}^{n} ; \mathbf{R}^{n}\right)$ and that the set $M_{\vec{a}}=\left\{x \in \mathbf{R}^{n}\right.$; $\vec{a}(x)=0\}$ has measure zero. Then

$$
(H(\lambda \vec{a})+1)^{-1} \rightarrow 0 \text { strongly, as } \lambda \rightarrow \infty .
$$

Proof. By our assumptions, $C_{c}^{\infty}\left(\Omega_{\vec{a}}\right)$ is dense in $L_{2}\left(\mathbf{R}^{n}\right)$, where $\Omega_{\vec{a}}=M_{\vec{a}}^{C}$. Given $\psi \in C_{c}^{\infty}\left(\Omega_{\vec{a}}\right)$, there is a $\delta>0$ so that $|\vec{a}(x)|>\delta$ on the support of $\psi$. We then have (treating $\vec{p}=-i \nabla$ as a perturbation of $\lambda \vec{a}$ in the second resolvent equation)

$$
\begin{aligned}
& \left.\|\left((\vec{p}-\lambda \vec{a})^{2}+1\right)^{-1}-\left((\lambda \vec{a})^{2}+1\right)^{-1}\right) \psi \| \\
& \leqq\left\|\left(\vec{p}^{2}-\lambda \vec{p} \cdot \vec{a}-\lambda \vec{a} \cdot \vec{p}\right)\left((\lambda \vec{a})^{2}+1\right)^{-1} \psi\right\| \\
& \leqq\left\|\left[\Delta\left((\lambda \vec{a})^{2}+1\right)^{-1}\right] \psi\right\|+2\left\|\left[\nabla\left((\lambda \vec{a})^{2}+1\right)^{-1}\right] \cdot \nabla \psi\right\| \\
& +\left\|\left((\lambda \vec{a})^{2}+1\right)^{-1} \Delta \psi\right\|+\left\|\lambda[\nabla \cdot \vec{a}]\left((\lambda \vec{a})^{2}+1\right)^{-1} \psi\right\| \\
& +2\left\|\lambda \vec{a} \cdot\left[\nabla\left((\lambda \vec{a})^{2}+1\right)^{-1}\right] \psi\right\|+2\left\|\lambda \vec{a} \cdot\left((\lambda \vec{a})^{2}+1\right)^{-1} \nabla \psi\right\| \\
& \leqq c|\lambda|^{-1}
\end{aligned}
$$

here [.] denotes multiplication by the function inside the square brackets.

The above result may seem somewhat disturbing because it shows that the resolvent of $H(\lambda \vec{a})$ will in some cases strongly converge to zero even in regions where the field is zero. A typical example for this phenomenon is given by the case where the vector potential is constant, but non-zero. Here, of course, one might object that we have chosen the "wrong" gauge. A less trivial example is provided by a magnetic field that is constant in a cylinder in $\mathbf{R}^{3}$. To avoid smoothness problems, we actually assume that the field is given by a smooth non-negative function $\varphi(r)$ of $r=\sqrt{x^{2}+y^{2}}$, with $0 \leqq \varphi \in C_{c}^{\infty}(\mathbf{R}), \varphi(t)=1$, for $0 \leqq t \leqq 1$. In a radial gauge, the associated vector potential is then of the form $(-y a(r), x a(r), 0)$, where $a$ solves the o. d. e. $r a^{\prime}(r)+2 a(r)=\varphi(r)$, and satisfies $a(r)=\frac{1}{2}$, for $0 \leqq r \leqq 1$. It is easily seen that $a$ is non-zero a. e., and hence the strong resolvent limit of $H(\lambda \vec{a})$ will be zero. Note that in this example there is no hope to find a gauge with the property that the vector field is zero outside the cylinder because integrating the vector field along a closed loop around the cylinder must give the total magnetic flux, which, however, is non-zero. (That this has physical consequences in quantum mechanics is just the Bohm-Aharonov effect.) Hence there is no chance of finding a limiting operator with Dirichlet boundary conditions on the cylinder.

To get a better understanding of what is going on here, we think classically: as a first approximation an operator (an observable) is a function on phase space. The correspondence is roughly that given by a pseudodifferential operator and its symbol. Intuitively, a sequence of operators $A_{n}(x, D)$ should converge strongly to zero as $n \rightarrow \infty$ if the symbols $A_{n}(x, \xi)$ are uniformly bounded and converge to zero uniformly for $(x, \xi)$ in any compact subset of phase space. Again, intuitively, $A_{n}(x, D)$ should converge to zero in norm if $A_{n}(x, \xi) \rightarrow 0$ uniformly on all of phase space. This kind of intuition makes Theorem 2.1 sound very reasonable. 
An even rougher intuition might suggest the following. Defining $\Omega_{\vec{a}}=\{x$ : $\vec{a}(x) \neq 0\}$ we have

$$
\lim _{\lambda \rightarrow \infty}\left[(\xi-\lambda \vec{a}(x))^{2}+1\right]^{-1}=\left\{\begin{array}{ll}
0 ; & x \in \Omega_{\vec{a}} \\
\left(\xi^{2}+1\right)^{-1} ; & x \in \Omega_{\vec{a}}^{C}
\end{array} .\right.
$$

It should therefore be true that

$$
\left((\vec{p}-\lambda \vec{a})^{2}+1\right)^{-1} \rightarrow\left(-\Delta_{\Omega_{\vec{a}}^{C}}+1\right)^{-1}, \quad \text { strongly . }
$$

But this intuition is incorrect because of non-local effects: if $\vec{a} \in C^{2}\left(\mathbf{R}^{n} ; \mathbf{R}^{n}\right)$ the proof of Theorem 2.1 shows that

$$
\left((\vec{p}-\lambda \vec{a})^{2}+1\right)^{-1} \chi_{\Omega_{\vec{a}}} \rightarrow 0, \quad \text { strongly , }
$$

but it does not follow that

$$
\chi_{\Omega_{\vec{a}}}\left((\vec{p}-\lambda \vec{a})^{2}+1\right)^{-1} \chi_{\Omega_{\vec{a}}^{C}} \rightarrow 0 \quad \text { strongly. }
$$

(This quantity would be identically zero classically, where all operators commute.) For an example, take $n=1$ and $a \in C_{0}^{\infty}((0,1))$ with $a(x)$ not identically zero in $(0,1)$. Then, if $f^{\prime}(x)=a(x)$ and $f(x)=0$, for $x \geqq 1$,

$$
\begin{aligned}
\varphi_{\lambda}(x): & =\left(\left((p-\lambda a)^{2}+1\right)^{-1} \psi\right)(x) \\
& =\frac{1}{2} \mathrm{e}^{i \lambda f(x)} \int \mathrm{e}^{-|x-y|} \mathrm{e}^{-i \lambda f(y)} \psi(y) d y,
\end{aligned}
$$

so if $\psi \in C_{0}^{\infty}((1, \infty))$ and $x<1$,

$$
\varphi_{\lambda}(x)=\frac{1}{2} \mathrm{e}^{-i \lambda \int_{x}^{1} a(t) d t} \mathrm{e}^{x} \int \mathrm{e}^{-y} \psi(y) d y
$$

which shows that $\chi_{\Omega_{\vec{a}}}\left((p-\lambda a)^{2}+1\right)^{-1} \chi_{\Omega_{\vec{a}}^{c}}$ does not converge strongly. Here of course we have a pure gauge transformation, and if the example is any guide it points to the necessity of having $B=d \vec{a}$ non-zero in $\Omega_{\vec{a}}$ if $\lambda \rightarrow \infty$ is to result in Dirichlet boundary conditions. (Note that the resolvent does not even converge weakly in this example.)

In contrast, we will now obtain strong resolvent convergence to the Dirichlet Laplacian on $M=\{x ; d \vec{a}(x)=0\}$ if the measure of the set $M \backslash M_{\vec{a}}$ is zero.

2.2. Theorem. Let $\vec{a} \in C^{1}\left(\mathbf{R}^{n} ; \mathbf{R}^{n}\right)$ and suppose that meas $\left(M \backslash M_{\vec{a}}\right)=0$, where $M=$ $\{x ; d \vec{a}(x)=0\}$ and $M_{\vec{a}}=\{x ; \vec{a}(x)=0\}$. Let $-\Delta_{M}$ denote the Dirichlet Laplacian on $M$, as defined above. Then

$$
H(\lambda \vec{a}) \rightarrow-\Delta_{M}, \text { in strong resolvent sense, } \lambda \rightarrow \infty .
$$

In fact, the resolvents converge uniformly in the gauge in the sense that for any $\varphi \in L_{2}\left(\mathbf{R}^{n}\right)$,

$$
\lim _{\lambda \rightarrow \infty} \sup _{U \in \mathscr{G}}\left\|U\left[(H(\lambda \vec{a})+1)^{-1}-\left(-\Delta_{M}+1\right)^{-1}\right] U^{-1} \varphi\right\|=0,
$$

where $\mathscr{G}$ is the subgroup of unitary operators consisting of gauge transformations, $U \varphi(x)=e^{l f(x)} \varphi(x)$, with $f$ real-valued. 
The underlying mechanism which produces Dirichlet boundary conditions is nonclassical in the sense that it stems from the non-commutativity of $x$ and $\vec{p}$ (cf. Proposition 1.4). This mechanism results in the type of convergence given by Eq. (2.1), which is midway between strong and uniform: it is uniform in the momentum, though perhaps not in position. In particular, one can take $f(x)=c \cdot x$ so that $U \vec{p} U^{-1}=\vec{p}-c$ in (2.1). We point out for comparison's sake that $\left\|A_{n}\right\| \rightarrow 0$ is equivalent to

$$
\lim _{n \rightarrow \infty} \sup _{U \in \mathscr{U}}\left\|U A_{n} U^{-1} \varphi\right\|=0
$$

for some non-zero $\varphi \in L^{2}$, where $\mathscr{U}$ is the group of all unitaries.

In Sect. 4, we will discuss a kind of converse result; in fact, Theorem 4.1 states that strong resolvent convergence, uniform in the gauge, can only hold if $M \backslash M_{\vec{a}}$ has empty interior.

In addition to Lemmas 1.1-1.3, the proof of Theorem 2.2 requires two more preparatory lemmas, which will also be of use in Sect. 3. We begin with a basic lemma which transforms a (local) lower bound on the magnetic field into a resolvent estimate.

2.3. Lemma. Let $\vec{a} \in C^{1}\left(\mathbf{R}^{n}, \mathbf{R}^{n}\right)$. Let $\mathcal{O} \subset \mathscr{U}$ be open sets in $\mathbf{R}^{n}$ with dist $(\mathcal{O}, \partial \mathscr{U})$ $>0$, and suppose that there exists a constant $b>0$ such that

$$
(\psi, H(\lambda \vec{a}) \psi) \geqq \lambda b\|\psi\|^{2}, \quad \lambda>0,
$$

for all $\psi \in C_{c}^{\infty}(\mathscr{U})$. Then there exists a constant $c$, depending on $\mathcal{O}, \mathscr{U}$ and $b$, such that

$$
\| H(\lambda \vec{a})+1)^{-1} \chi_{\mathcal{O}} \| \leqq c \lambda^{-1 / 2}, \quad \lambda>0 .
$$

Proof. We can find (non-negative) functions $\eta_{1}, \eta_{2} \in C^{\infty}\left(\mathbf{R}^{n}\right)$ such that $\eta_{1}^{2}+\eta_{2}^{2}=$ 1 , with $\eta_{1}(x)=1$ for $x \in \mathcal{O}$ and supp $\eta_{1} \subset \mathscr{U}$. In addition, we may assume that $\left|\nabla \eta_{1}\right|,\left|\nabla \eta_{2}\right|$ are bounded functions. Then

$$
H(\lambda \vec{a})=\eta_{1} H(\lambda \vec{a}) \eta_{1}+\eta_{2} H(\lambda \vec{a}) \eta_{2}-\left|\nabla \eta_{1}\right|^{2}-\left|\nabla \eta_{2}\right|^{2}
$$

By assumption, we have

$$
\eta_{1} H(\lambda \vec{a}) \eta_{1} \geqq \lambda b \eta_{1}^{2} \geqq \lambda b \chi_{0},
$$

and we can find a constant $c_{0}$ such that $H(\lambda \vec{a}) \geqq \lambda b \chi_{\mathcal{O}}-c_{0}$; without loss, we may assume $c_{0} \geqq 1$. It follows that

$$
\left(H(\lambda \vec{a})+c_{0}\right)^{-1 / 2} \chi_{\mathcal{O}}\left(H(\lambda \vec{a})+c_{0}\right)^{-1 / 2} \leqq(\lambda b)^{-1},
$$

so that

$$
\left\|\left(H(\lambda \vec{a})+c_{0}\right)^{-1} \chi_{\mathcal{O}}\right\| \leqq\left\|\left(H(\lambda \vec{a})+c_{0}\right)^{-1 / 2} \chi_{\mathcal{O}}\right\| \leqq(\lambda b)^{-1 / 2} .
$$

Finally, by the resolvent equation,

$$
\left\|(H(\lambda \vec{a})+1)^{-1} \chi_{\mathcal{O}}\right\| \leqq c_{0}(\lambda b)^{-1 / 2},
$$

and the desired estimate follows.

In the following lemma we show that we may "enhance" the convergence properties of $H(\lambda \vec{a})$ by adding in a term $n(\lambda) \chi_{\Omega_{n(\lambda)}}$. 
2.4. Lemma. Let $\vec{a} \in C^{1}\left(\mathbf{R}^{n} ; \mathbf{R}^{n}\right)$. Suppose $\left\{\Omega_{n}\right\}$ is an increasing sequence of open subsets of $\mathbf{R}^{n}$ with the property that for each $n$ we have an estimate

$$
\left\|(H(\lambda \vec{a})+1)^{-1} \chi \Omega_{n}\right\| \leqq c_{n} \lambda^{-1 / 2}, \quad \lambda>0,
$$

for some constant $c_{n}$.

Then there exists a function $n:[0, \infty) \rightarrow \mathbf{N}$ with $n(\lambda) \rightarrow \infty$, as $\lambda \rightarrow \infty$, such that

$$
\left\|\left(H(\lambda \vec{a})+n(\lambda) \chi_{\Omega_{n(i)}}+1\right)^{-1}-(H(\lambda \vec{a})+1)^{-1}\right\| \rightarrow 0, \quad \lambda \rightarrow \infty,
$$

and, for any $t \geqq 0$,

$$
\left\|e^{-t\left(H(\lambda \vec{a})+n(\lambda) \chi_{\Omega_{n(j)}}\right)}-e^{-t H(\lambda \vec{a})}\right\| \rightarrow 0, \quad \lambda \rightarrow \infty .
$$

Proof. We first note that we may assume $c_{n+1} \geqq c_{n}$ so that $n^{2} c_{n}$ is increasing. Defining $n(\lambda)$ to be the largest integer $n$ such that

$$
n^{2} c_{n} \leqq \lambda^{1 / 2}
$$

we see that $n(\lambda) \rightarrow \infty$, as $\lambda \rightarrow \infty$, and

$$
\begin{aligned}
& \left\|\left(H(\lambda \vec{a})+n(\lambda) \chi_{\Omega_{n(\lambda)}}+1\right)^{-1}-(H(\lambda \vec{a})+1)^{-1}\right\| \\
& \quad \leqq\left\|n(\lambda) \chi_{\Omega_{n(\lambda)}}(H(\lambda \vec{a})+1)^{-1}\right\| \leqq n(\lambda) c_{n(\lambda)} \lambda^{-1 / 2} \leqq 1 / n(\lambda),
\end{aligned}
$$

proving the first statement.

It follows that for any continuous function $f:[0, \infty) \rightarrow \mathbf{C}$ with $f(\eta) \rightarrow 0$, as $\eta \rightarrow \infty$, we have

$$
\left\|f\left(H(\lambda \vec{a})+n(\lambda) \chi_{\Omega_{n(\lambda)}}+1\right)-f(H(\lambda \vec{a})+1)\right\| \rightarrow 0, \quad \lambda \rightarrow \infty,
$$

and the second statement follows.

We are now ready for the proof of Theorem 2.2 .

Proof of Theorem 2.2. (1) We first choose a sequence of open sets $\Omega_{n} \uparrow \Omega=M^{C}$ with $\bar{\Omega}_{n}$ compact and $\bar{\Omega}_{n} \subset \Omega$.

Let $x_{0} \in \bar{\Omega}_{n}$. Then there exist indices $i, j$ such that $B_{l j}\left(x_{0}\right) \neq 0$. Without restriction, we may assume that there exists an open set $\mathscr{W} \subset \Omega$ such that $x_{0} \in \mathscr{W}$ and $B_{i j}(x) \geqq b>0$, for $x \in \mathscr{W}$. Then, if $\mathcal{O} \subset \subset \mathscr{W}$ and $x_{0} \in \mathcal{O}$, the estimate of [AHS; Thm. 2.9], reproduced in Proposition 2.4 above, implies that

$$
(\psi, H(\lambda \vec{a}) \psi) \geqq \lambda b\|\psi\|^{2}, \quad \psi \in C_{c}^{\infty}(\mathscr{W}),
$$

and Lemma 2.3 yields

$$
\left\|(H(\lambda \vec{a})+1)^{-1} \chi_{\mathscr{O}}\right\| \leqq c_{\mathscr{O}} \lambda^{-1 / 2}, \quad \lambda>0 .
$$

By a simple compactness argument, we find that there exist constants $c_{n}>0$ such that

$$
\left\|(H(\lambda \vec{a})+1)^{-1} \chi_{\Omega_{n}}\right\| \leqq c_{n} \lambda^{-1 / 2}, \quad \lambda>0,
$$

and we may apply Lemma 2.4 to obtain a function $n(\lambda) \uparrow \infty$ such that, for any $t \geqq 0$,

$$
\left\|\mathrm{e}^{-t\left(H(\lambda \vec{a})+n(\lambda) \chi_{\left.\Omega_{n(\lambda)}\right)}\right)}-\mathrm{e}^{-t H(\lambda \vec{a})}\right\| \rightarrow 0, \quad \lambda \rightarrow \infty .
$$


(2) We will now show that, for any $f \in C_{c}^{\infty}\left(\mathbf{R}^{n}\right)$ and $t>0$,

$$
\left(\mathrm{e}^{-t H(\lambda \vec{a})}-\mathrm{e}^{t \Delta_{M}}\right) f \rightarrow 0, \quad \lambda \rightarrow \infty .
$$

From (2.4) the claimed strong resolvent convergence then follows via the Laplace transform. Now we have (writing $\chi_{n(\lambda)}=\chi_{\Omega_{n(\lambda)}}$ )

$$
\begin{aligned}
\left\|\left(\mathrm{e}^{-t H(\lambda \vec{a})}-\mathrm{e}^{t \Delta_{M}}\right) f\right\| \leqq & \left\|\left(\mathrm{e}^{-t(H(\lambda \vec{a})}-\mathrm{e}^{-t\left(H(\lambda \vec{a})+n(\lambda) \chi_{n(\lambda)}\right)}\right) f\right\| \\
& +\|\left(\mathrm{e}^{-t\left(H(\lambda \vec{a})+n(\lambda) \chi_{n(\lambda)}-\mathrm{e}^{t \Delta_{M}}\right) f \| .} .\right.
\end{aligned}
$$

For fixed $t>0$, the first term on the RHS tends to 0 , as $\lambda \rightarrow \infty$, by Eq. (2.3), while the second term on the RHS goes to 0 by Lemmas 1.1 and 1.3 , and (2.4) follows.

(3) The proof of Eq. (2.1) is rather simple. We just note that the only place where strong convergence rather than uniform convergence is used in the above arguments is when Lemma 1.1 and Lemma 1.3 come into play. Here it is crucial that we have the absolute value of $f$ in the RHS of the inequality in Lemma 1.3.

\section{Norm Resolvent Convergence}

In this section we first derive a fundamental criterion which reduces the convergence problem for $H(\lambda \vec{a})$, as $\lambda \rightarrow \infty$, to the more standard convergence problem for $-\Delta+n \chi_{n}$, as $n \rightarrow \infty$, where the $\chi_{n}$ are certain characteristic functions. We then discuss various applications of our criterion.

3.1. Main Lemma. Let $\vec{a} \in C^{1}\left(\mathbf{R}^{n} ; \mathbf{R}^{n}\right), M=\{x ; d \vec{a}(x)=0\}, M_{\vec{a}}=\{x ; \vec{a}(x)=0\}$ and suppose that meas $\left(M \backslash M_{\vec{a}}\right)=0$. Let $-\Delta_{M}$ denote the Dirichlet Laplacian on $M$, as defined above. Suppose there exists a sequence of open sets $\Omega_{n} \subset \Omega=M^{C}$ such that $\Omega_{n} \uparrow \Omega$, satisfying the following two conditions:

(i) There exist constants $c_{n}>0$ such that

$$
\left\|(H(\lambda \vec{a})+1)^{-1} \chi_{\Omega_{n}}\right\| \leqq c_{n} \lambda^{-1 / 2}, \quad \lambda>0 .
$$

(ii) As $n \rightarrow \infty,-\Delta+n \chi_{\Omega_{n}}$ converges to $-\Delta_{M}$ in norm resolvent sense.

Then

$$
H(\lambda \vec{a}) \rightarrow-\Delta_{M} \quad \text { in norm resolvent sense, } \lambda \rightarrow \infty .
$$

Remarks (a) In our applications, we will use Lemma 2.3 to verify condition (i).

(b) Conditions (i) and (ii) are in competition for the size of the sets $\Omega_{n}$ : while condition (i) is easier to satisfy for smaller $\Omega_{n}$, we cannot hope for norm convergence in (ii) if the sets $\Omega_{n}$ are too small.

Proof of Lemma 3.1. The proof is very similar to the second part of the proof of Theorem 2.2. Again, from the estimate (3.1) and Lemma 2.4 it follows that there exists a function $n(\lambda):[0, \infty) \rightarrow \mathbf{N}$ with $n(\lambda) \rightarrow \infty$ as $\lambda \rightarrow \infty$ such that (again, $\left.\chi_{n(\lambda)}=\chi_{\Omega_{n(\lambda)}}\right)$

$$
\left\|\mathrm{e}^{-t\left(H(\lambda \vec{a})+n(\lambda) \chi_{n(i)}\right)}-\mathrm{e}^{-t H(\lambda \vec{a})}\right\| \rightarrow 0, \quad \lambda \rightarrow \infty,
$$


and we may estimate, for any $t>0$,

$$
\begin{aligned}
\left\|\mathrm{e}^{-t H(\lambda \vec{a})}-\mathrm{e}^{t \Delta_{M}}\right\| \leqq & \left\|\mathrm{e}^{-t H(\lambda \vec{a})}-e^{-t\left(H(\lambda \vec{a})+n(\lambda) \chi_{n(\lambda)}\right)}\right\| \\
& +\left\|\mathrm{e}^{-t\left(H(\lambda \vec{a})+n(\lambda) \chi_{n(\lambda,)}\right)}-\mathrm{e}^{t \Delta_{M}}\right\| .
\end{aligned}
$$

Here the first term on the RHS goes to 0 as $\lambda \rightarrow \infty$, by Eq. (3.2), while the second term on the RHS tends to 0 by assumption (ii), Lemma 1.3, and [RSI; Theorem VIII.20]. Thus we have shown that, for any $t>0$,

$$
\left\|\mathrm{e}^{-t H(\lambda \vec{a})}-\mathrm{e}^{t \Delta_{M}}\right\| \rightarrow 0, \quad \lambda \rightarrow \infty .
$$

Hence, by the Laplace transform and dominated convergence,

$$
\left\|(H(\lambda \vec{a})+1)^{-1}-\left(-\Delta_{M}+1\right)^{-1}\right\| \leqq \int_{0}^{\infty}\left\|e^{-t H(\lambda \vec{a})}-\mathrm{e}^{t \Delta_{M}}\right\| \mathrm{e}^{-t} d t
$$

tends to zero, as $\lambda \rightarrow \infty$.

The following proposition will be one of our main tools in checking condition (ii) of Lemma 3.1.

3.2. Proposition. Suppose $\Omega_{n} \uparrow \Omega$, where $\Omega_{n}, \Omega$ are open sets in $\mathbf{R}^{n}$, and suppose that $\bar{\Omega} \backslash \Omega_{1}$ is compact. Let $M=\Omega^{C}$. Then

$$
-\Delta+n \chi_{\Omega_{n}} \rightarrow-\Delta_{M} \text { in norm resolvent sense, } \quad n \rightarrow \infty
$$

Proof. Choose an open set $U \supset \bar{\Omega} \backslash \Omega_{1} \supset \partial \Omega$ with $\bar{U}$ compact, and a non-negative $\varphi \in C_{c}^{\infty}\left(\mathbf{R}^{n}\right)$ with $\varphi=1$ on $U$. Then with

$$
R_{n}=\left(-\Delta+n \chi_{\Omega_{n}}+1\right)^{-1}, \quad R_{0}=(-\Delta+1)^{-1}, \quad R=\left(-\Delta_{M}+1\right)^{-1},
$$

and $\varphi$ denoting multiplication by the function $\varphi$, we have

$$
0 \leqq \varphi\left(R_{n}-R\right) \varphi \leqq \varphi\left(R_{0}-R\right) \varphi
$$

with the last operator compact. Since $\varphi\left(R_{n}-R\right) \varphi \downarrow 0$ it follows that ([K; Thm. VIII-3.5])

$$
\left\|\varphi\left(R_{n}-R\right) \varphi\right\|=\left\|\left(R_{n}-R\right)^{1 / 2} \varphi\right\|^{2} \rightarrow 0
$$

and thus

$$
\left\|\left(R_{n}-R\right) \varphi\right\|=\left\|\left(R_{n}-R\right)^{1 / 2}\left(R_{n}-R\right)^{1 / 2} \varphi\right\| \rightarrow 0 .
$$

As in the proof of Lemma 2.3, we have

$$
\left\|R_{n} \chi_{\Omega_{n}}\right\| \leqq n^{-1 / 2}
$$

so

$$
\left\|\left(R_{n}-R\right) \chi_{\Omega_{1}}\right\| \rightarrow 0 .
$$

Let $\psi=(1-\varphi) \chi_{\Omega^{C}}$ and note that $\psi \in C^{\infty}$. We calculate

$$
\begin{aligned}
(-\Delta+1)\left[\psi\left(R_{0}-R\right)\right] & =(-\Delta \psi)\left(R_{0}-R\right)-2(\nabla \psi) \cdot \nabla\left(R_{0}-R\right) \\
& =(\Delta \varphi) \chi_{\Omega^{C}}\left(R_{0}-R\right)+2(\nabla \varphi) \chi_{\Omega^{C}} \cdot \nabla\left(R_{0}-R\right),
\end{aligned}
$$


so that

$$
\psi\left(R_{0}-R\right)=R_{0} \chi_{\Omega^{C}}(\Delta \varphi)\left(R_{0}-R\right)+2 R_{0} \chi_{\Omega^{C}}(\nabla \varphi) \cdot \nabla\left(R_{0}-R\right)
$$

is a compact operator. Thus $\psi\left(R_{n}-R\right) \psi \leqq \psi\left(R_{0}-R\right) \psi$ implies

$$
\left\|\left(R_{n}-R\right)(1-\varphi) \chi_{\Omega^{C}}\right\| \rightarrow 0 \text {. }
$$

But

$$
\begin{aligned}
R_{n}-R= & \chi_{\Omega_{1}}\left(R_{n}-R\right)+\chi_{\Omega \backslash \Omega_{1}} \varphi\left(R_{n}-R\right)+\chi_{\Omega^{C}} \varphi\left(R_{n}-R\right) \\
& +\chi_{\Omega^{C}}(1-\varphi)\left(R_{n}-R\right),
\end{aligned}
$$

and it follows that $\left\|R_{n}-R\right\| \rightarrow 0$.

Our first corollory deals with the case of compact $\bar{\Omega}$.

3.3. Corollary. Let $\vec{a} \in C^{1}\left(\mathbf{R}^{n} ; \mathbf{R}^{n}\right)$ and $\Omega_{\vec{a}}=\{x ; \vec{a}(x) \neq 0\}, \Omega=\{x ; d \vec{a}(x) \neq 0\}$, $M=\Omega^{C}$. Suppose $\Omega_{\vec{a}} \backslash \Omega$ has measure zero and $\bar{\Omega}$ is compact. Then

$$
H(\lambda \vec{a}) \rightarrow-\Delta_{M} \text { in norm resolvent sense }, \quad \lambda \rightarrow \infty .
$$

Proof. Let $\Omega_{n} \uparrow \Omega$ with $\bar{\Omega}_{n} \subset \Omega$. We need only check that condition ( $i$ ) of Lemma 3.1 is satisfied. But this follows from a simple compactness argument as in the proof of Theorem 2.2.

We next consider the half-space problem in situations where in any left halfspace of the form $\left\{x ; x_{1}<-\delta\right\}$ there is a uniform (positive) lower bound for the strength of the magnetic field (or rather a positive lower bound for the quadratic form $(\psi, H(\lambda \vec{a}) \psi))$, while the vector potential is identically zero on the half-space $x_{1} \geqq 0$. Again, we wish to obtain norm resolvent convergence to the Dirichlet Laplacian living on the set $x_{1}>0$.

3.4. Corollary. Suppose $\vec{a} \in C^{1}\left(\mathbf{R}^{n}, \mathbf{R}^{n}\right)$ vanishes on the half-space $\left\{x \in \mathbf{R}^{n}\right.$; $\left.x_{1}>0\right\}$. Assume that for any $\delta>0$ there is a positive constant $c_{\delta}$ such that

$$
(\psi, H(\lambda \vec{a}) \psi) \geqq c_{\delta} \lambda\|\psi\|^{2}, \quad \psi \in C_{c}^{\infty}\left(\left\{x ; x_{1}<-\delta\right\}\right), \lambda>0 .
$$

Then $H(\lambda \vec{a}) \rightarrow-\Delta_{+}$in norm resolvent sense, where $-\Delta_{+}$denotes the Dirichlet Laplacian on the half-space $\left\{x ; x_{1}>0\right\}$.

Remark. It seems to be rather difficult to find a most general condition in terms of the coefficients $B_{l j}(x)$ of the magnetic field $d \vec{a}$ which would guarantee condition (3.3) to hold. Going back to Lemma 2.3 and its proof, one can see that it is not enough to simply require a lower bound $\sum\left|B_{i j}(x)\right| \geqq \delta_{n}>0$, for $x \in \Omega_{n}=$ $\left\{x ; x_{1}<-1 / n\right\}$. In addition, one needs some uniformity concerning the balls where a lower bound holds for some specific $\left|B_{i j}(x)\right|$.

To be more precise, it would be sufficient to require that for any $n \in \mathbf{N}$, there exist some $\delta_{n}>0, \varrho_{n}>0$, and a covering of $\Omega_{n+1}$ with a sequence of balls, $B_{k}$, of radius $\varrho_{n}$ which enjoys the following two properties:

(i) there exists a $K$ such that at most $K$ members of the family $\left\{B_{k}\right\}$ intersect any given ball of radius 1 .

(ii) for each of the balls $B_{k}$, we can find a pair of indices $i, j$ such that $\left|B_{\imath j}(x)\right| \geqq$ $\delta_{n}$, for $x \in B_{k}$. 
Then, using an IMS-partition of unity ([CFrKS]) consisting of functions $\psi_{k} \in$ $C_{c}^{\infty}\left(B_{k}\right)$ with $\sum \psi_{k}^{2}(x)=1$, for $x \in \Omega_{n}$, and $\sup _{k}\left\|\nabla \psi_{k}\right\|_{\infty}<\infty$, we can follow the line of proof used in proving Lemma 2.3 to derive the desired lower bound.

Proof of Corollary 3.4. Let $\Omega_{n}=\left\{x \in \mathbf{R}^{n} ; x_{1}<-1 / n\right\}$. Then we clearly need only show that the operators $-\Delta+n \chi_{\Omega_{n}}$ converge to $-\Delta_{+}$in norm resolvent sense as $n \rightarrow \infty$. By a partial Fourier transform in the $n-1$ variables orthogonal to the $x_{1}$-direction we need only show that with

$$
h_{+}(k)=-d^{2} / d x^{2}+|k|^{2} \text { on }(0, \infty)
$$

with a Dirichlet boundary condition at the point 0 , for $k \in \mathbf{R}^{n-1}$, and

$$
h_{n}(k)=-d^{2} / d x^{2}+n \chi_{(-\infty,-1 / n)}(x)+|k|^{2}
$$

on the real line, we have

$$
\left\|\left(h_{+}(k)+1\right)^{-1}-\left(h_{n}(k)+1\right)^{-1}\right\| \rightarrow 0, \quad n \rightarrow \infty,
$$

uniformly in $k$. But for large $|k|$ the difference is small uniformly in $n$ so that we need only prove (3.4) for $k=0$ (note that this automatically gives uniformity of the convergence for $k$ in compacts). It follows from Proposition 3.2 (in one dimension) that

$$
\left\|\left(h_{n}(0)+1\right)^{-1}-R\right\| \rightarrow 0,
$$

where $R$ is the resolvent of $\left(-d^{2} / d x^{2}\right)_{0}$, the 1-dimensional Laplace operator on the closed set $[0, \infty)$, defined as in Sect. 2 . But it is very easy to see that this is just the usual Dirichlet Laplacian on $(0, \infty)$.

Remark. One might conjecture - based on Corollary 3.4 and Proposition 3.2 - that more generally there is norm resolvent convergence as long as there is no problem getting a lower bound near the boundary of $\Omega$. This is incorrect as the next example shows.

3.5. Example. Let $\Omega \subset \mathbf{R}^{2}$ be defined as $\Omega=\left\{x \in \mathbf{R}^{2} ; x_{2}<\left|x_{1}\right|^{-1}\right\}$. Letting $M=$ $\Omega^{C}$, we have $-\Delta+\lambda \chi_{\Omega} \rightarrow-\Delta_{M}, \lambda \rightarrow \infty$, in strong resolvent sense, but not in norm resolvent sense.

Proof. The claimed strong resolvent convergence is immediate from Lemma 1.1. Assume now for a contradiction that

$$
\left\|\left(-\Delta+\lambda \chi_{\Omega}+1\right)^{-1}-\left(-\Delta_{M}+1\right)^{-1}\right\| \rightarrow 0, \quad \lambda \rightarrow \infty .
$$

We consider a sequence of functions $f_{n}, n=0,1,2, \ldots$, with $f_{n}$ given as the characteristic function of the ball of radius 1 centered at the point $(0, n)$ in the plane, and we let

$$
u_{n}=(-\Delta+1)^{-1} f_{n}, \quad v_{n}=\left(-\Delta_{M}+1\right)^{-1} f_{n}, \quad w_{n, \lambda}=\left(-\Delta+\lambda \chi_{\Omega}+1\right)^{-1} f_{n} .
$$

We will first show that the assumed norm resolvent convergence implies that

$$
\left\|v_{n}-u_{n}\right\| \rightarrow 0, \quad n \rightarrow \infty
$$


In fact, if the convergence of the resolvents would be uniform on the unit ball of $L_{2}\left(\mathbf{R}^{n}\right)$, then, given $\varepsilon>0$, we could find $\lambda_{\varepsilon} \geqq 0$ such that

$$
\left\|v_{n}-w_{n, \lambda}\right\|<\varepsilon, \quad \lambda \geqq \lambda_{\varepsilon} \quad n \in \mathbf{N} .
$$

Furthermore, for any fixed $\lambda \geqq 0$, we have

$$
\left\|u_{n}-w_{n, \lambda}\right\| \rightarrow 0, \quad n \rightarrow \infty
$$

In order to prove (3.7), we translate by $-n$ along the $x_{2}$-axis, i.e., we compare $u_{0}=(-\Delta+1)^{-1} f_{0}$ with

$$
\tilde{w}_{n, \lambda}=\left(-\Delta+\lambda \chi \Omega_{n}+1\right)^{-1} f_{0}
$$

with $\Omega_{n}=\left\{\left(x_{1}, x_{2}\right) \in \mathbf{R}^{2} ;\left(x_{1}, x_{2}+n\right) \in \Omega\right\}$, so that, in particular, $\left\|u_{n}-w_{n, \lambda}\right\|=$ $\left\|u_{0}-\tilde{w}_{n, \lambda}\right\|$. By monotone convergence of quadratic forms, the resolvent of $-\Delta+$ $\lambda \chi_{\Omega_{n}}$ converges strongly to the resolvent of $-\Delta$, as $n \rightarrow \infty$, and we see that $u_{0}-\tilde{w}_{n, \lambda} \rightarrow 0$, proving (3.7).

Now (3.5) is immediate from Eqs. (3.6) and (3.7).

On the other hand, it can be shown that

$$
\liminf _{n \rightarrow \infty}\left\|v_{n}-u_{n}\right\|>0
$$

in contradiction with $(3.5)$.

It remains to prove (3.8). Here we introduce, finally, $z_{n}=\left(-\left(\Delta_{-} \oplus \Delta_{+}\right)\right.$ $+1)^{-1} f_{n}$, where $-\Delta_{ \pm}$is the Dirichlet Laplacian on the left- and on the right half plane, respectively. By translation invariance with respect to the $x_{2}$-coordinate, we clearly have $\left\|u_{n}-z_{n}\right\|=c>0$, for a suitable constant $c$. On the other hand, $\left\|v_{n}-z_{n}\right\| \rightarrow 0, n \rightarrow \infty$, which may be seen as follows:

Again, we translate by $-n$ the $x_{2}$-direction and prove that $\left\|\tilde{v}_{n}-z_{0}\right\| \rightarrow 0, n \rightarrow$ $\infty$, where $\tilde{v}_{n}=\left(-\Delta_{M_{n}}+1\right)^{-1} f_{0}$, and $M_{n}=\Omega_{n}^{C}$. The quadratic forms $\mathbf{t}_{n}$, associated with $-\Delta_{M_{n}}$, have form domain $\mathscr{2}\left(\mathbf{t}_{n}\right)=\mathscr{H}^{1}\left(M_{n}\right)$, with $M_{n} \subset M_{n+1}$, for all $n$. Hence the sequence $\left(\mathbf{t}_{n}\right)$ is monotonically decreasing and the standard convergence theorem tells us that the strong resolvent limit of the operators $-\Delta_{M_{n}}$ is given as the selfadjoint and non-negative operator representing the closed quadratic form $\int|\nabla \varphi|^{2} d x$ with domain given as the closure of $\cup \mathscr{Q}\left(\mathbf{t}_{n}\right)$ in the $\|\cdot\|_{1,2}$-norm which is easily seen to coincide with the form domain of $-\left(\Delta_{-} \oplus \Delta_{+}\right)$.

3.6. Remark. While our standard assumption is to have $\vec{a}$ of class $C^{1}$, in many cases our approach would also work under weaker regularity assumptions. For example, let us consider the case where we have a constant, non-zero magnetic field on the left half-plane and zero field on the right half-plane in $\mathbf{R}^{2}$, with coordinates $x$ and $y$, and a vector potential $\vec{a}$ given as

$$
\vec{a}=\vec{a}(x, y)=(0, B \bar{x}),
$$

where $B$ is a positive constant and $\bar{x}=x$, if $x \leqq 0$, and $\bar{x}=0$, if $x>0$. Here one can directly prove that

$$
(\varphi, H(\lambda \vec{a}) \varphi) \geqq \lambda B \int_{\{(x, y) ; x<0\}}|\varphi|^{2} d x d y, \quad \varphi \in C_{c}^{\infty}\left(\mathbf{R}^{2}\right) .
$$


The proof of Eq. (3.9) is analogous to the proof of Proposition 2.4 ([AHS; Thm. $2.9])$, but some more care is needed in proving the commutation relation: As $\vec{a}=$ $\left(a_{1}(x, y), a_{2}(x, y)\right)$ with $a_{1}=0$ and $a_{2}=B \bar{x}$, we have

$$
\left(\left[\left(p_{1}-a_{1}\right),\left(p_{2}-a_{2}\right)\right] \varphi, \varphi\right)=\left(\left[-i \partial_{x}, \bar{x}\right] \varphi, \varphi\right)=-i B \int_{\{x<0\}}|\varphi|^{2} d x d y
$$

by a partial integration in the $x$-variable (in fact, in 1 dimension,

$$
\left.\left(-\varphi^{\prime}, \bar{x} \varphi\right)+\left(\bar{x} \varphi,-\varphi^{\prime}\right)=-\int_{-\infty}^{0} \bar{\varphi}^{\prime} x \varphi-\int_{-\infty}^{0} \bar{\varphi} x \varphi^{\prime}=\int_{-\infty}^{0}|\varphi|^{2} .\right)
$$

From Lemma 2.3 and the lower bound given in Eq. (3.9) we may now infer that

$$
\left\|(H(\lambda \vec{a})+1)^{-1} \chi_{\{x<0\}}\right\| \leqq \lambda^{-1 / 2} .
$$

Therefore, using either Lemma 3.1 directly or the ideas used in its proof, we finally see that we only have to make sure that

$$
\left\|\left(-d^{2} / d x^{2}+n \chi_{-}(x)+k^{2}+1\right)^{-1}-\left(h_{+}(k)+1\right)^{-1}\right\| \rightarrow 0, \quad n \rightarrow \infty,
$$

uniformly in $k$. This, however, is essentially the same expression we have dealt with in the proof of Corollary 3.4, and we may conclude that $H(\lambda \vec{a})$ converges to $-\Delta_{+}$in norm resolvent sense, as $\lambda \rightarrow \infty$.

Our next aim is to show that we have norm resolvent convergence if the magnetic field $B=d \vec{a}$ is periodic with respect to some discrete lattice and satisfies the condition that meas $\left(M \backslash M_{\vec{a}}\right)=0$. Here we first prove an auxiliary result which may also be of use in situations where one does not have periodicity; e. g., one could base an alternative proof of Corollary 3.4 on Proposition 3.7.

3.7. Proposition. Let $\Psi_{m}$ denote the characteristic function of the standard unit cube centered at $m \in \mathbf{Z}^{n}$. Suppose $\left\{A_{n}\right\}$ is a sequence of bounded self-adjoint operators satisfying the following two conditions:

(a) for any $r>0$, we have

$$
\sup \left\{\left\|\Psi_{m} A_{n} \Psi_{m^{\prime}}\right\| ; m, m^{\prime} \in \mathbf{Z}^{n},\left|m-m^{\prime}\right|<r\right\} \rightarrow 0, \quad n \rightarrow \infty,
$$

and

Then $\left\|A_{n}\right\| \rightarrow 0$, as $n \rightarrow \infty$.

$$
\sup _{n} \sup _{m \in \mathbf{Z}^{n}} \sum_{\left\{m^{\prime} ;\left|m-m^{\prime}\right| \geqq r\right\}}\left\|\Psi_{m} A_{n} \Psi_{m^{\prime}}\right\| \rightarrow 0, \quad r \rightarrow \infty .
$$

Proof. Given $\varepsilon>0$, we will show that, for $n$ large enough, the norm of

$$
A_{n}^{(L)}=\sum_{|m| \leqq L,\left|m^{\prime}\right| \leqq L} \Psi_{m} A_{n} \Psi_{m^{\prime}}
$$

is smaller than $\varepsilon$, uniformly in $L$. Now choose $r$ large enough to ensure that the expression in (b) is smaller than $\varepsilon / 2$. Then, by Schur's inequality, the norm of

$$
A_{n, 2}^{(L)}=\sum_{\left|m-m^{\prime}\right| \geqq r} \Psi_{m} A_{n}^{(L)} \Psi_{m^{\prime}}
$$


is less than $\varepsilon / 2$, for all $n, L$. Let $c_{r}=\#\left\{m^{\prime} ;\left|m^{\prime}\right| \leqq r\right\}$. Then the number of terms appearing in the expansion of $\left(A_{n, 1}^{(L)}\right)^{\ell}=\left(A_{n}^{(L)}-A_{n, 2}^{(L)}\right)^{\ell}$ as a sum of terms of the form

$$
\Psi_{m_{1}} A_{n}^{(L)} \Psi_{m_{2}} A_{n}^{(L)} \Psi_{m_{3}} \cdot \ldots \cdot A_{n}^{(L)} \Psi_{m_{\ell+1}}
$$

is bounded by $C_{L} \cdot r^{\ell}$. Thus if we choose $N$ such that $\left\|\Psi_{m} A_{n} \Psi_{m^{\prime}}\right\|<\varepsilon / 2 c_{r}$, for $\left|m-m^{\prime}\right|<r$ and $n \geqq N$, we then have

$$
\left\|\left(A_{n, 1}^{(L)}\right)^{\ell}\right\| \leqq\left(\frac{\varepsilon}{2 c_{r}}\right)^{\ell} c_{r}^{\ell} C_{L}
$$

This gives, for $n \geqq N$ and for all $\ell \in \mathbf{N}$,

$$
\left\|A_{n, 1}^{(L)}\right\| \leqq \frac{\varepsilon}{2} L^{1 / \ell}
$$

which implies $\left\|A_{n, 1}^{(L)}\right\| \leqq \varepsilon / 2$, for all $n \geqq N$ and all $L$. Thus $\left\|A_{n}^{(L)}\right\|<\varepsilon$, and the proof is complete.

The following simple lemma assures that condition (b) of Proposition 3.7 is satisfied under very general assumptions.

3.8. Lemma. Let $V \geqq 0$ be locally integrable, and let $-\Delta+V$ be defined via quadratic forms. Then

$$
\sup _{m \in \mathbf{Z}^{n}} \sum_{\left\{m^{\prime} ;\left|m-m^{\prime}\right| \geqq r\right\}}\left\|\Psi_{m}(-\Delta+V+1)^{-1} \Psi_{m^{\prime}}\right\| \rightarrow 0, \quad r \rightarrow \infty,
$$

uniformly for all $V$ satisfying the above conditions.

Proof. As $V \geqq 0$, we have the fundamental estimate (cf., e. g., Simon [S3])

$$
\left|(-\Delta+V+1)^{-1} f\right| \leqq(-\Delta+1)^{-1}|f|, \text { a. e , }
$$

for $f \in \mathscr{H}$. Furthermore, it is well-known that there exist constants $C$ and $\alpha>0$ such that

$$
\left\|\Psi_{k}(-\Delta+1)^{-1} \Psi_{j}\right\| \leqq C \mathrm{e}^{-\alpha|k-j|}, \quad k, j \in \mathbf{Z}^{n}
$$

this can be shown, e. g., by direct calculation starting from the heat kernel and using the Laplace transform. It follows that

$$
\left\|\Psi_{m}(-\Delta+V+1)^{-1} \Psi_{m^{\prime}} f\right\| \leqq\left\|\Psi_{m}(-\Delta+1)^{-1} \Psi_{m^{\prime}}|f|\right\| \leqq C e^{-\alpha\left|m-m^{\prime}\right|}\|f\| .
$$

Now the proof is easily completed by an elementary estimate.

It is now easy to obtain the following result on norm resolvent convergence for periodic magnetic fields:

3.9. Theorem. Let $\vec{a} \in C^{1}\left(\mathbf{R}^{n}, \mathbf{R}^{n}\right)$, and suppose that the associated field $B=d \vec{a}$ is periodic with respect to the lattice $\mathbf{Z}^{n}$. Assume, furthermore, that meas $\left(M \backslash M_{\vec{a}}\right)=$ 0 , where $M=\{x ; d \vec{a}(x)=0\}$ and $M_{\vec{a}}=\{x ; \vec{a}(x)=0\}$. Then

$$
H(\lambda \vec{a}) \rightarrow-\Delta_{M} \text { in norm resolvent sense, } \lambda \rightarrow \infty,
$$

where $-\Delta_{M}$ denotes the Dirichlet Laplacian of the set $M$. 
Proof. We begin the proof by choosing an increasing sequence of open sets $\Omega_{n} \subset$ $\Omega=M^{C}$, where we assume that each $\Omega_{n}$ is periodic (i. e., invariant under lattice translations), $\bar{\Omega}_{n} \subset \Omega$ and $\cup \Omega_{n}=\Omega$. Then condition $(i)$ in the Main Lemma 3.1 is satisfied as may be seen again from the estimate given in Proposition 1.4, Lemma 2.3 and a simple compactness argument (using also the periodicity of the sets $\Omega_{n}$ ).

For condition (ii) in the Main Lemma, we apply Proposition 3.7 with

$$
A_{n}=\left(-\Delta+n \chi_{\Omega_{n}}+1\right)^{-1}-\left(-\Delta_{M}+1\right)^{-1} \text {. }
$$

Note first that condition (b) of Proposition 3.7 is satisfied by Lemma 3.8, and it only remains to check condition (a), where, by periodicity, it is enough to consider $m=0 \in \mathbf{Z}^{n}$. Now, for $r \in \mathbf{N}$ fixed, and $\Xi_{r}=\sum_{\left|m^{\prime}\right|<r} \Psi_{m^{\prime}}$, we have

$$
\begin{gathered}
\sup \left\{\left\|\Psi_{0}\left(\left(-\Delta+n \chi_{\Omega_{n}}+1\right)^{-1}-\left(-\Delta_{M}+1\right)^{-1}\right) \Psi_{m^{\prime}}\right\| ; m^{\prime} \in \mathbf{Z}^{d},\left|m^{\prime}\right|<r\right\} \\
\leqq\left\|\Xi_{r}\left(\left(-\Delta+n \chi_{\Omega_{n}}+1\right)^{-1}-\left(-\Delta_{M}+1\right)^{-1}\right) \Xi_{r}\right\| .
\end{gathered}
$$

Here the difference of resolvents on the RHS converges strongly to zero by Lemma 1.1. Since the operator $\Xi_{r}(-\Delta+1)^{-1} \Xi_{r}$ is compact and the operators on the RHS form a monotonic sequence, it follows that the RHS tends to zero, as $n \rightarrow \infty$, and we are done.

Since norm resolvent convergence implies convergence of spectra in the sense of [RSIV; Sect. VIII], we immediately obtain the following corollary:

3.10. Corollary. Suppose that with $B=d \vec{a}$ periodic as in Theorem 3.9 , we have

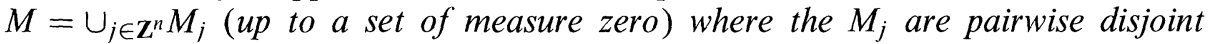
compact sets with $M_{j}=M_{0}+j$. Assume that the interior of $M$ is non-empty and let $\left\{\mu_{k} ; k=1,2, \ldots\right\}$ be the eigenvalues of $-\Delta_{M_{0}}$. Finally, fix $k, a, b, c$ satisfying $\mu_{k}<a<b<\mu_{k+1}<c$.

Then for large enough $\lambda$,

$$
\begin{gathered}
\sigma(H(\lambda \vec{a})) \cap[a, b]=\emptyset, \\
\sigma(H(\lambda \vec{a})) \cap[b, c] \neq \emptyset .
\end{gathered}
$$

Proof. It is easy to see that $\Delta_{M}=\oplus_{j \in \mathbf{Z}^{n}} \Delta_{M_{j}}$ with form-domain $\oplus \mathscr{H}^{1}\left(M_{j}\right)$. Thus the spectrum of $-\Delta_{M}$ is the same as that of $-\Delta_{M_{0}}$, but with infinite multiplicity. The statements (3.10) and (3.11) are standard consequences of norm resolvent convergence.

Remark. (a) Colloquially speaking one might say that, as $\lambda \rightarrow \infty$, gaps will open up in the spectrum of $H(\lambda \vec{a})$, while the spectrum of $H(\lambda \vec{a})$ concentrates around the eigenvalues of $-\Delta_{M}$. As we will explain below, we can not prove, however, that the spectrum of $H(\lambda \vec{a})$ will always consist of non-trivial intervals.

(b) As was noted in the introduction, it is a classical result that periodic Schrödinger operators $-\Delta+V$ may have spectral gaps, in any dimension. While, in 1-dimension, there will generically be an infinite number of gaps (Simon [S1]), one expects at most a finite number of gaps in dimension $\geqq 2$; see, e. g., Skriganov [Skr] or Karpeshina [Kar]. Similarly, one-dimensional Dirac operators with periodic potentials generically have an infinite number of gaps; see Schmidt [Schm]. For Laplace-Beltrami operators defined by a periodic metric tensor over $\mathbf{R}^{n}$, Davies and 
Harrell $[\mathrm{DH}]$ have constructed examples in dimensions 2 and higher with spectral gaps (in 1-dim., any Laplace-Beltrami operator of this type is equivalent to $-d^{2} / d x^{2}$ on the real line, and there are no gaps). Finally, in connection with a problem in control theory (cf. Avellaneda et al. [AADH]), Hempel [He] has produced examples of periodic elliptic divergence type operators $-\sum_{i j} \partial_{j} a_{i j}(x) \partial_{i}$, acting in $L_{2}\left(\mathbf{R}^{n}\right)$, with spectral gaps; this again works for all dimensions.

In situations which are much more general than what we consider here and which include the case of periodic magnetic fields, Brüning and Sunada ([BrS1, BrS2]) show that the spectrum of the associated operator can be written as the union of mutually disjoint closed intervals such that any compact subset of $\mathbf{R}$ meets only finitely many. (This does not imply the existence of spectral gaps, however.)

We now illustrate Corollary 3.10 by a simple example of a periodic magnetic Schrödinger operator with spectral gaps, in any dimension $\geqq 2$. Here both $\vec{a}$ and $B$ will be periodic.

3.11. Example. Let $\varphi \in C^{\infty}(\mathbf{R})$ be periodic with period 1 , and assume that $\varphi(t)=$ 0 , for $-1 / 4 \leqq t \leqq 1 / 4$. Furthermore, let us suppose that $\varphi^{\prime}(t) \neq 0$, for almost every $t \in(1 / 4,3 / 4)$. We then define a vector potential $\vec{a} \in C^{\infty}\left(\mathbf{R}^{n} ; \mathbf{R}^{n}\right)$, by setting

$$
\vec{a}\left(x_{1}, \ldots, x_{n}\right)=\left(\varphi\left(x_{n}\right), \varphi\left(x_{1}\right), \ldots, \varphi\left(x_{n-1}\right)\right) ;
$$

it is clear that $\vec{a}$ is periodic with respect to the lattice $\mathbf{Z}^{n}$. Up to a set of measure zero, the region $M_{\vec{a}}$ is then given by the union of $Q_{0}+j, j \in \mathbf{Z}^{n}$, where $Q_{0}$ is the standard cube of sidelength $1 / 2$ centered at the origin. In order to be able to apply Corollary 3.10, we only have to make sure that $M \backslash M_{\vec{a}}$ has measure zero. Letting $\omega(x)=\varphi\left(x_{n}\right) d x_{1}+\varphi\left(x_{1}\right) d x_{2}+\ldots+\varphi\left(x_{n-1}\right) d x_{n}$ denote the 1-form associated with $\vec{a}$, the magnetic field $B=d \omega$ is easily computed to be

$$
\begin{aligned}
B(x)= & \varphi^{\prime}\left(x_{n}\right) d x_{n} \wedge d x_{1}+\varphi^{\prime}\left(x_{1}\right) d x_{1} \wedge d x_{2}+\varphi^{\prime}\left(x_{2}\right) d x_{2} \wedge d x_{3} \\
& +\ldots+\varphi^{\prime}\left(x_{n-1}\right) d x_{n-1} \wedge d x_{n} .
\end{aligned}
$$

For $n \geqq 3$, it is evident that $d \omega(x) \neq 0$, for almost every $x \in M_{\vec{a}}$.

For $n=2$, however, the magnetic field is given by the function $B\left(x_{1}, x_{2}\right)=$ $\varphi^{\prime}\left(x_{2}\right)-\varphi^{\prime}\left(x_{1}\right)$ and we make the additional assumption that $\varphi^{\prime \prime}$ is non-zero almost everywhere in $(1 / 4,3 / 4)$. Then $\nabla B\left(x_{1}, x_{2}\right)=\left(-\varphi^{\prime \prime}\left(x_{1}\right), \varphi^{\prime \prime}\left(x_{2}\right)\right)$ is non-zero almost everywhere in $\Omega_{\vec{a}}$ so the same holds for $B$.

Therefore our example satisfies all the assumptions of Corollary 3.10, and it follows that, as $\lambda$ increases, the spectrum of $H(\lambda \vec{a})$ will converge to the spectrum of $-\Delta_{Q_{0}}$.

Note that in the 2-dimensional case the total magnetic flux through a period cell $Q$ is zero, if $\vec{a}$ is periodic. This follows from Stokes' Theorem and the observation that the line integral $\vec{a} \cdot d l$ over $\partial Q$ vanishes because of the periodicity of $\vec{a}$. This underlines again that our result is very much different from the well-known fact that a non-zero constant magnetic field in 2 dimensions leads to spectral gaps.

It has been one of our main goals to produce spectral gaps of $H(\lambda \vec{a})$ (with periodic vector potential $\vec{a}$ or periodic field $B$ ), and we have achieved this goal by now, but one may still wonder whether the spectrum separating the gaps will consist of closed intervals of positive length, or whether it may collapse into isolated points. This is a rather delicate question in the case of periodic fields since a non-zero constant field in two dimensions leads to spectrum consisting of isolated 
eigenvalues. Of course, this does not imply anything about the spectrum for nonconstant periodic fields.

We now consider the case of periodic vector potentials $\vec{a}$, where Floquet theory yields a direct fiber decomposition of the operator. In particular, the spectrum has band structure since it can be written as the union of the ranges of the "bandfunctions," a countable family of continuous functions defined on a fundamental cell of the dual lattice. Clearly, if we could show that the spectrum of $H(\lambda \vec{a})$ is purely absolutely continuous, then the non-collapse of the bands would immediately follow (although results weaker than absolute continuity may also be sufficient; cf. e. g., the result on line broadening announced in Iwatsuka [Iw; Theorem 4.6]). In view of the work of Gérard [G] on the band functions of periodic Hamiltonians $-\Delta+V$, we expect that $H(\vec{a})$ has absolutely continuous spectrum except for a possible discrete set of eigenvalues. We exclude this possibility at least for small $\vec{a}$.

3.12. Theorem. Suppose $\left\{\eta_{j}\right\}_{j=1}^{n}$ is a basis for $\mathbf{R}^{n}$. Then there is an $\alpha>0$ so that for any $\vec{a} \in C^{1}\left(\mathbf{R}^{n} ; \mathbf{R}^{n}\right)$ which is periodic in the sense that $\vec{a}\left(x+\eta_{j}\right)=\vec{a}(x), j=$ $1, \ldots, n$, the self-adjoint operator

$$
H(\vec{a})=(\vec{p}-\vec{a})^{2}
$$

has purely absolutely continuous spectrum if

$$
\sup _{x}|\vec{a}(x)|<\alpha .
$$

Proof. We adapt Thomas' proof [Th] of the absolute continuity for periodic Schrödinger operators, $-\Delta+V$, as reproduced in [RSIV, p. 309]: There is a unitary operator $U: L^{2}\left(\mathbf{R}^{n}\right) \rightarrow \mathscr{H}$,

$$
\mathscr{H}=\int_{\tilde{Q}}^{\oplus} \ell^{2}\left(\mathbf{Z}^{n}\right) d^{n} k,
$$

where $\tilde{Q}=\left\{\Sigma t_{j} K_{j} ; 0 \leqq t_{j}<1, j=1, \ldots, n\right\}$, and $\left\{K_{j}\right\}_{j=1}^{n}$ is the basis dual to $\left\{\eta_{j}\right\}_{j=1}^{n}$,

Under this transformation we have

$$
\eta_{j} \cdot K_{\ell}=2 \pi \delta_{j \ell} .
$$

$$
\left(U(-i \nabla) U^{-1} g\right)(k)_{m}=(P(k) g(k))_{m},
$$

where

$$
(P(k) g(k))_{m}=\left(k+\Sigma m_{j} K_{j}\right) g(k)_{m} .
$$

In addition,

$$
\left(U \vec{a} U^{-1} g\right)(k)_{m}=(\tilde{\vec{a}} g(k))_{m},
$$

where

$$
(\tilde{\vec{a} g} g))_{m}=\sum_{\beta \in \mathbf{Z}^{n}} \hat{\vec{a}}_{\beta} g(k)_{m-\beta},
$$

and

$$
\hat{\vec{a}}_{m}=(\operatorname{vol} Q)^{-1} \int_{Q} e^{\left(-i \sum_{J=1}^{n} m_{J} K_{J} \cdot x\right)} \vec{a}(x) d^{n} x
$$

with

$$
Q=\left\{\Sigma t_{j} \eta_{j} ; 0 \leqq t_{j}<1, j=1, \ldots, n\right\}
$$


It suffices to show (see [RSIV, p. 309]) that for each $d \in \mathbf{R}^{n}$, the operator

$$
H(z)=(P(k)-\tilde{\vec{a}})^{2} ; \quad k=d+z b, \quad b=\eta_{1}
$$

cannot have the same real eigenvalue $C$ for all $z \in \mathbf{C}$.

As in [RSIV] we see that with $H_{0}(z)=P(k)^{2}, z=\lambda+i y$ with $\lambda$ chosen so that $b \cdot(d+\lambda b)=\pi$, we have

$$
\lim _{y \rightarrow \infty}\left\|\left(H_{0}(z)+1\right)^{-1}\right\|=0 .
$$

It follows by the resolvent equation that if

$$
\liminf _{y \rightarrow \infty}\left\|\left(H(z)-H_{0}(z)\right)\left(H_{0}(z)+1\right)^{-1}\right\|<1
$$

then for a sequence of $y^{\prime} s \rightarrow \infty$ we have

$$
\left\|(H(z)+1)^{-1}\right\| \rightarrow 0,
$$

which contradicts the existence of a fixed eigenvalue $(C+1)^{-1}$ of $(H(z)+1)^{-1}$. We see that

$$
\lim _{y \rightarrow \infty}\left\|\left((\tilde{\vec{a}})^{2}+i(\nabla \cdot a)^{-}\right)\left(H_{0}(z)+1\right)^{-1}\right\|=0
$$

where $(\nabla \cdot \vec{a})^{\sim}$ is defined analogously to $\tilde{\vec{a}}$. It remains to estimate

$$
\liminf _{y \rightarrow \infty}\left\|2 \tilde{\vec{a}} \cdot P(k)\left(P(k)^{2}+1\right)^{-1}\right\|,
$$

where $k=d+z b$. This is easily done. We find that

$$
\begin{aligned}
\xi(d) & =\liminf _{y \rightarrow \infty}\left\||P(k)|\left(P(k)^{2}+1\right)^{-1}\right\| \\
& =\xi(0)<\infty,
\end{aligned}
$$

so that if $2 \sup _{x}|\vec{a}(x)| \xi(0)<1$, there is no constant eigenvalue. Thus we can take $\alpha=(2 \xi(0))^{-1}$.

\section{Gauge Invariance and the Condition $S_{\vec{a}}$.}

We have isolated a condition on the supports of $\vec{a}(x)$ and $B(x)=d \vec{a}(x)$, namely that the set $M \backslash M_{\vec{a}}$ have measure zero, which we have called Condition $S_{\vec{a}}$. (Here, again, $M=\{x ; B(x)=0\}, \Omega=M^{C}$, and $\left.M_{\vec{a}}=\{x ; \vec{a}(x)=0\}, \Omega_{\vec{a}}=M_{\vec{a}}^{C}\right)$. Under condition $S_{\vec{a}}$ we have shown in Theorem 2.2 that $H(\lambda \vec{a})$ converges in the strong resolvent sense, uniformly in the gauge, to $-\Delta_{M}$. That something like condition $S_{\vec{a}}$ is natural is shown in the following theorem.

4.1. Theorem. Let $\vec{a} \in C^{2}\left(\mathbf{R}^{n}, \mathbf{R}^{n}\right)$, and suppose that $M \backslash M_{\vec{a}}=\Omega_{\vec{a}} \backslash \Omega$ has nonempty interior. Then there is no bounded operator $L$ so that $(H(\lambda \vec{a})+1)^{-1}$ converges to $L$ strongly, uniformly in the gauge.

Proof. Suppose the contrary. As in the proof of Theorem 2.1 we show that, as $\lambda \rightarrow \infty$,

$$
(H(\lambda \vec{a})+1)^{-1} \chi_{\Omega_{\vec{a}}} \rightarrow 0, \quad \text { strongly. }
$$

Thus $L \chi_{\Omega_{\vec{a}}}=0$. Choose an open ball $B_{0}$ such that $\bar{B}_{0} \subset \Omega_{\vec{a}} \backslash \Omega$ and a $g \in C^{3}\left(\mathbf{R}^{n}\right)$ so that $\tilde{\vec{a}}=\vec{a}+d g=0$ in $B_{0}$. The function $g$ can be obtained in the following way: Letting $B_{1}$ denote an open ball satisfying $\bar{B}_{0} \subset B_{1}$ and $\bar{B}_{1} \subset \Omega_{\vec{a}} \backslash \Omega$, we first construct a function $g_{1}$ in $B_{1}$ by writing $g_{1}(x)$ as a line integral of $\vec{a}$ along a smooth path $\Gamma_{x}$ from the center of $B_{1}$ to the point $x \in B_{1}$; we may then take $g=\eta g_{1}$, 
where $\eta \in C_{c}^{\infty}\left(B_{1}\right)$ satisfies $\eta(x)=1$, for $x \in B_{0}$. Choose $0 \neq f \in C_{c}^{\infty}\left(B_{0}\right)$ and let $\psi=(-\Delta+1) f$. Then

$$
\left((H(\lambda \tilde{\vec{a}})+1)^{-1}-e^{-i \lambda g} L e^{i \lambda g}\right) \psi \rightarrow 0, \quad \lambda \rightarrow \infty,
$$

since we have assumed that $(H(\lambda \tilde{\vec{a}})+1)^{-1}$ converges to $L$ strongly, uniformly in the gauge, and thus

$$
(H(\lambda \tilde{\vec{a}})+1)^{-1} \psi \rightarrow 0, \quad \lambda \rightarrow \infty .
$$

But $\psi=(-\Delta+1) f=(H(\lambda \tilde{\vec{a}})+1) f$, so $(H(\lambda \tilde{\vec{a}})+1)^{-1} \psi=f$ is constant.

We should not expect Condition $S_{\vec{a}}$ to be gauge invariant. We have chosen a gauge in writing $-\Delta_{M}$ and thus we should expect that we need to choose a gauge for the operators which tend to $-\Delta_{M}$. However, the following (gauge invariant) condition on the field $B$ is an interesting one to consider.

We say that a 2-form $B$ satisfies Condition $S_{B}$, if there exists a vector potential, $\vec{a}$, such that $B=d \vec{a}$ and $M \backslash M_{\vec{a}}$ has measure zero.

(In this context, we always assume that $B=d \vec{a}$ for some $\vec{a}$ or, equivalently, that $d B=0$.) Of course, if Condition $S_{B}$ is true then we can find a gauge so that $S_{\vec{a}}$ is true, and then the main theorems of this paper apply. We will now try to establish that Condition $S_{B}$ is (roughly) equivalent to a zero-flux condition:

Let $M^{0}$ denote the interior of $M$. It is clear from Stokes' Theorem that if $B$ satisfies Condition $S_{B}$, then the flux of $B$ through any (smooth and oriented) 2surface $D$, with (smooth and oriented) boundary $\partial D$ contained in $M^{0}$, is zero,

$$
\text { Flux of } B \text { through } D=\int_{D} d \vec{a}=\int_{\partial D} \vec{a}=0,
$$

where $\vec{a}$ is as in Condition $S_{B}$; again, we think of $\vec{a}$ as a 1 -form. It is an interesting topological and analytical question whether a zero-flux condition of this type on the field $B$ is in some sense sufficient for the validity of Condition $S_{B}$. The following argument is not a proof but indicates how a proof may be constructed in certain situations: assuming the zero-flux condition and $B=d \vec{a}$, we try to find an $f$ such that $\tilde{\vec{a}}=\vec{a}-d f$ satisfies $S_{\tilde{a}}$. Proceeding as in the proof of Theorem 4.1 , we first define $f$ in each component of $M^{0}$ by a line integral of $\vec{a}$ along a path in this component starting from some reference point and going to the point $x$. Under the zero-flux condition, $f(x)$ is well-defined in $M^{0}$. Then we extend $f$ to all of $\mathbf{R}^{n}$. Unfortunately, this extension process may be difficult or impossible in certain situations. It would be interesting to understand the phenomena which can occur in this context.

Acknowledgements. The authors are most grateful to T. Hoffmann-Ostenhof for his kind invitation to the Erwin Schrödinger Institute, where most of this work was done, stimulated by the strong coffee brewed at the ESI. They also thank B. Simon for helpful suggestions. Furthermore, Rainer Hempel would like to thank Evans Harrell (Georgia Tech) for several discussions on periodic operators.

\section{References}

[AB] Arendt, W., Batty, C.J.K.: Absorption semi-groups and Dirichlet boundary conditions. Math. Ann. 295, 427-448 (1993)

[AADH] Avellaneda, M., Alama, S., Deift, P.A., Hempel, R.: On the existence of eigenvalues of a divergence form operator $A+\lambda B$ in a gap of $\sigma(A)$. Asymptotic Analysis, to appear 
[AHS] Avron, J., Herbst, I., Simon, B.: Schrödinger operators with singular magnetic fields, I. General Interactions. Duke Math. J. 45, 847-883 (1978)

[BrS1] Brüning, J., Sunada, T.: On the spectrum of gauge-periodic elliptic operators. Soc. Math. France Astérisque 210, 65-74 (1992)

[BrS2] Brüning, J., Sunada, T.: In preparation

[CFrKS] Cycon, H.L., Froese, R., Kirsch, W., Simon, B.: Schrödinger operators. Berlin, Heidelberg, New York: Springer, 1987

[DH] Davies, E.B., Harrell, E.: Conformally flat Riemannian metrics, Schrödinger operators, and semiclassical approximation. J. Differential Equations 66, 165-188 (1987)

[GTr] Gilbarg, D., Trudinger, N.S.: Elliptic Partial Differential Equations of Second Order. Berlin, Heidelberg, New York: Springer, 1977

[G1] Gérard, Chr.: Resonance theory for periodic Schrödinger operators. Bull. Soc. Math. France 118, 27-54 (1990)

[H] Hedberg, L.: Spectral synthesis and stability in Sobolev spaces. In: Euclidean Harmonic Analysis, Proceedings Maryland. Lecture Notes in Mathematics 779, 73-103 (1980)

[He] Hempel, R.: Second order perturbations of divergence type operators with a spectral gap. In: Operator Theory: Advances and Applications, Vol. 47, Basel, Birkhäuser, 1992

[HZh] Herbst, I., Zhao, Zh.: Sobolev spaces, Kac-regularity, and the Feynman-Kac formula. In: Seminar on Stochastic Processes, Boston, Birkhäuser: 1987

[Iw] Iwatsuka, A.: On Schrödinger operators with magnetic fields. In: Lecture Notes in Mathematics, vol. 1450 Conf. Proc. Fujita, H., Ikebe, T., Kuroda, S.T. (eds.), Berlin, Heidelberg, New York: Springer 1990, pp.157-172

[Kar] Karpeshina, Yu.E.: Perturbation theory for the Schrödinger operator with a periodic potential. Proc. of the Steklov Inst. of Math. 1991, Issue 3, pp. 109-145

[K] Kato, T.: Perturbation theory for linear operators. Berlin, Heidelberg, New York: Springer 1966

[RSI] Reed, M., Simon, B.: Methods of Modern Mathematical Physics, Vol. I. Functional Analysis. Revised and enlarged edition. New York: Academic Press, 1979

[RSIV] Reed, M., Simon, B.: Methods of Modern Mathematical Physics, Vol. IV. Analysis of Operators. New York: Academic Press, 1978

[S1] Simon, B.: On the genericity of nonvanishing instability intervals in Hill's equation. Ann. Inst. H. Poincaré Sect. A (N. S.) 24, 91-93 (1976)

[S2] Simon, B.: A canonical decomposition for quadratic forms with applications to monotone convergence theorems. J. Funct. Anal. 28, 377-385 (1978)

[S3] Simon, B.: Functional Integration and Quantum Physics. New York: Academic Press, 1979

[Schm] Schmidt, K.M.: On the genericity of nonvanishing instability intervals in periodic Dirac systems. Ann. Inst. Henri Poincaré 59, 315-326 (1993)

[Skr] Skriganov, M.M.: The spectrum band structure of the three-dimensional Schrödinger operator with periodic potential. Inventiones Math. 80, 107-121 (1985)

[St] Stein, E.M.: Singular integrals and differentiability properties of functions. Princeton, NJ: Princeton, Univ. Press 1986

[Th] Thomas, L.E.: Time dependent approach to scattering from impurities in a crystal. Commun. Math. Phys. 33, 335-343 (1973)

[W] Weidmann, J.: Stetige Abhängigkeit der Eigenwerte und Eigenfunktionen elliptischer Differentialoperatoren vom Gebiet. Math. Scand. 54, 51-69 (1984)

Note added in proof. In the case of a Dirac operator with periodic electric potentials, L.I. Danilov has found results that are related to what we have stated in Theorem 3.12 (cf. L.I. Danilov, On the spectrum of the Dirac operator in $\mathbf{R}^{n}$ with periodic potential, Teoret. Mat. Fiz. 85 (1990), 41-53 (russian); translation in: Theoret. and Math. Phys. 85 (1991), 1039-1048). The authors thank Prof. M. Birman (St. Petersburg) for drawing their attention to this paper. 
